\title{
DISEASE COURSE IN EARLY RHEUMATOID ARTHRITIS: AN OBSERVATIONAL STUDY
}

\author{
Teodora Serban ${ }^{1,2}$, Iulia Satulu², Oana Vutcanu ${ }^{2}$, Mihaela Milicescu ${ }^{1,2}$, Carina Mihai ${ }^{1,2}$, \\ Mihai Bojinca ${ }^{1,2}$, Victor Stoica ${ }^{1,2}$ \\ ${ }^{1}$ Early Inflammatory Arthritis Center, Internal Medicine and Rheumatology Clinic, \\ Dr. Ion Cantacuzino Clinical Hospital, Bucharest \\ ${ }^{2}$ Carol Davila University of Medicine and Pharmacy, Bucharest
}

\begin{abstract}
Background. In rheumatoid arthritis (RA), prompt diagnosis and initiation of disease-modifying treatment during the first months after disease onset - a period called "window of opportunity" - is significantly superior to the delayed start of the same therapy. Clinical remission is more frequently obtained in patients with a disease duration no longer than 4 months and is the main aim of the treatment, therefore the "treat to target" (T2T) and "tight control" strategies were proposed.

Objective. The aim of this study is to evaluate the therapy used for patients with early RA (ERA) and the impact of this medication on the clinical outcomes at 12 months after the first evaluation.

Methods. Patients with early arthritis who were referred to the Early Arthritis Research Center of Dr. I. Cantacuzino Hospital between 2010-2014 and who fulfilled the 2010 EULAR/ACR Classification Criteria for RA (and who did not satisfy classification criteria for other inflammatory rheumatologic diseases) were enrolled. Only patients who received treatment with Methotrexate (MTX) associated or not with corticosteroids (CS) and patients who fulfilled the classification criteria for RA but did not received any DMARDs therapy were enrolled.

Results. Forty-three patients were enrolled in the study, $62.8 \%$ females, mean age $55.47 \pm 13.71$ years, median (interquartile range) DAS28 5.07 (4.31-5.60), SDAI 29.02 (20.92-34.61). At the first presentation, 40 patients $(93.0 \%)$ received treatment with Methotrexate (MTX) in doses ranging from $5 \mathrm{mg} / \mathrm{week}$ to $20 \mathrm{mg} / \mathrm{week}$, with a mean dose of $11.16 \pm 4.47 \mathrm{mg} /$ week, the most frequently used doses ranging from $10 \mathrm{mg} / \mathrm{week}$ to $15 \mathrm{mg} /$ week. 26 patients $(60.5 \%)$ received corticosteroids (CS), either oral or intra-articular. During the study both the total number of patients receiving MTX and the mean dose of MTX increased, while the number of patients receiving CS decreased and at the end of the study only low-dose oral CS were still administered in 8 patients. At 12 months, median (interquartile range) DAS 28 was 1.77 (1.43-3.16), SDAI 3.58 (2.32-11.82). The evolution under treatment assessed by DAS28 and SDAI wasn't significantly different between patients who received, at baseline, MTX in association with CS (mean value: $\triangle \mathrm{DAS} 28=-2.58 \pm 1.72, \triangle \mathrm{SDAl}=-20.44 \pm 16.49$ ) and those who received MTX monotherapy (mean value: $\triangle \mathrm{DAS} 28=-2.91 \pm 1.17, \triangle \mathrm{SDAl}=-21.80 \pm 9.89)(\mathrm{p}>0.05)$. There was no significant difference in change from baseline of DAS28 and SDAI at 12 months between patients who received low-dose oral CS and those who received intermittent intra-articular CS ( $p>0.05)$.

Conclusions. Treatment with MTX and/or CS led to clinical and laboratory improved outcomes at 12 months of follow-up. There was no significant difference regarding long-term outcomes between patients who received lowdose oral CS and those who received intra-articular CS. As this study was performed on a relatively small number of real-life patients with ERA, the results obtained should be validated on larger cohorts of patients.
\end{abstract}

Keywords: early rheumatoid arthritis, treatment, "treat to target", clinical remission

\section{BACKGROUND}

Rheumatoid arthritis (RA) is an important problem of public health, due to the disability that it may induce and the high costs resulting from work absence and expensive treatments. The early period of RA, known as "window of opportunity", is a very short period of time in which initiation of diseasemodifying therapy improves the long-term out- comes. The cut-off for the duration of the "window of opportunity" is often reported to be 3 months or 12 weeks, but this is mainly based on expert opinion than on scientific data. $(1,2)$

In 2013, Smolen et al. published the EULAR recommendations for the management of RA. They stated that disease-modifying anti-rheumatic drug (DMARD) therapy should be started as soon as the

\footnotetext{
Correspondence address:

Teodora Serban, MD, PhD. Dr. Ion Cantacuzino Clinical Hospital, 5-7 Ion Movilă Street, Bucharest

E-mail: teodoramazdrag@yahoo.com
} 
diagnosis of RA is established and that Methotrexate (MTX) should be part of the initial treatment strategy. It is also stated that corticosteroids (CS) should be considered as part of the initial strategy of treatment, in combination with one or more DMARDs, for at most 6 months. (3)

In 2015, the ACR Guidelines for treatment of Rheumatoid Arthritis were published, including two separate sections: one for early RA (ERA) and one for established RA. For ERA patients, MTX monotherapy is considered the first option, while corticosteroids (CS) are recommended only if disease flares, for short periods of time and at the lowest effective dose. (4)

Clinical remission is the capital aim of the treatment of RA and therefore the "treat to target" (T2T) and "tight control" strategies were proposed. $(5,6)$

In 2012, the EULAR/ACR remission criteria for RA were proposed and they define remission by the Boolean-based definition: "At any time point, a patient must satisfy all of the following: $\mathrm{TJC} \leq 1, \mathrm{SJC} \leq 1$, $\mathrm{CRP} \leq 1 \mathrm{mg} / \mathrm{dl}$ and patient global assessment (PGA) $\leq 1$ (on a $0-10$ scale)" OR by the Index-based definition: "At any time point, a patient must have $\mathrm{SDAI} \leq 4$ ”. (7)

All these efforts of the experts' community underline the necessity to diagnose and treat RA very early, thus allowing patients to obtain and maintain remission and, on the long term, radiographic nonprogression and good physical function. In our Early Inflammatory Arthritis (EIA) Research Center, we strive to reach all these goals.

\section{OBJECTIVE}

The aim of this study was to evaluate the therapy used for patients with ERA attending our EIA center and its impact on the clinical outcomes at 12 months after the first presentation.

\section{METHODS}

All patients with EIA who were referred to our Early Inflammatory Arthritis center between 2010 2014 and who fulfilled the 2010 EULAR/ACR Classification Criteria for RA (8) were enrolled. Only patients who were naïve to DMARD therapy were enrolled. The study was approved by the local ethics committee and all patients gave a written informed consent. All patients were evaluated at baseline and after 12 months. Treatment was prescribed by an experienced rheumatologist according to the current guidelines, but not following a strict strategy. MTX was considered the DMARD treatment of choice taking into consideration the EULAR 2013 and ACR 2015 recommendations for the management of RA. Oral low-dose CS were administered in patients with polyarticular forms of presentation, while intra-articular CS were administered in patients with 1 to 3 joints with signs of inflammation. Clinical evaluation of the patients included monitoring of the number of tender joints (NTJ), number of swollen joints (NSJ), morning stiffness, and global assessment of disease activity on a $0-100 \mathrm{~mm}$ visual analogue scale (VAS) by the patient (PtGA) and by the investigator (PhGA). Laboratory assessments at every visit included acute phase reactants (C reactive protein, CRP and erythrocyte sedimentation rate, ESR) and immunologic markers (rheumatoid factor, RF and anti-citrullinated protein antibodies, ACPA). Disease activity was assessed by composite indices such as DAS28 (9) and SDAI (10). DAS28 was used to evaluate disease activity, due to the fact that this is the most frequently used tool in clinical practice, while comparison with SDAI was performed because the latter is considered a more stringent tool to evaluate disease activity, being also incorporated in the "Boolean-based definition" of remission. Statistical analysis was performed the software IBM SPSS 20.0. Numeric variables were described by means \pm standard deviations (SD), as well as medians and interquartile range (IQR) and comparisons between groups were performed by the Wilcoxon signed-rank test. To evaluate the value of different baseline parameters as predictors of remission at 12 months (assessed by DAS28 and SDAI) we used univariable logistic regression. Results were considered statistically significant if $\mathrm{p}$ values were $<0.05$.

\section{RESULTS}

\section{First presentation}

Forty-three patients were enrolled in the study, $62.8 \%$ female, mean age $55.47 \pm 13.71$ years. The median and interquartile range (IQR) for duration of symptoms before the first presentation were 2.00 months (1-3 months). Thirty-four (79.1\%) patients presented with polyarticular involvement. Patients positive for RF and ACPA were $90.7 \%$ and $90.7 \%$, respectively.

As expected, all the clinical and laboratory parameters reflecting disease activity (NTJ, NSJ, PtGA, PhGA, morning stiffness duration, ESR, CRP, 
DAS28, SDAI) decreased during the study and the difference was significant $(p<0.001)$. Median values and interquartile range (IQR) of the clinical and laboratory data of the study group at baseline and after 12 months are shown in Table 1.

TABLE 1. Clinical and laboratory parameters in 43 patients with ERA, at their first presentation in our EIA Research Center baseline and after 12 months

\begin{tabular}{|l|c|c|}
\hline Parameter & Baseline & 12 months \\
\hline NTJ (28 joints) & $10(5-13)$ & $0(0-4)$ \\
\hline NSJ (28 joints) & $4(2-7)$ & $0(0-0)$ \\
\hline $\begin{array}{l}\text { Morning } \\
\text { stiffness (min) }\end{array}$ & $60(45-90)$ & $15(5-30)$ \\
\hline $\begin{array}{l}\text { PtGA } \\
(0-100 ~ m m)\end{array}$ & $65(53-76)$ & $19(10-30)$ \\
\hline $\begin{array}{l}\text { PhGA } \\
(0-100 ~ m m)\end{array}$ & $50(40-60)$ & $10(10-20)$ \\
\hline CRP $(\mathrm{mg} / \mathrm{l})$ & $9.13(3.57-14.68)$ & $2.20(1.12-4.61)$ \\
\hline ESR $(\mathrm{mm} / \mathrm{h})$ & $27.00(14.00-51.00)$ & $14.00(10.00-22.00)$ \\
\hline DAS28 & $5.07(4.31-5.60)$ & $1.77(1.43-3.16)$ \\
\hline SDAl & $29.02(20.92-34.61)$ & $3.58(2.32-11.82)$ \\
\hline
\end{tabular}

NTJ = number of tender joints; NSL = number of swollen joints, PtGA and PhGA = global assessment of disease activity on 0-100 $\mathrm{mm}$ VAS, by the patients and by the physician, respectively; $\mathrm{CRP}=\mathrm{C}$-reactive protein; $\mathrm{ESR}=$ erythrocyte sedimentation rate; DAS28 = disease activity score 28; SDAI = simplified disease activity index.

\section{Comparison between DAS28 and SDAI}

At baseline, DAS28 identified 20 patients (46.5\%) as having medium disease activity and 21 patients $(48.8 \%)$ as having high disease activity, while SDAI identified 12 patients $(27.9 \%)$ as having medium disease activity and 29 patients $(67.3 \%)$ as having high disease activity. At 12 months, DAS28 identified 30 patients $(69.8 \%)$ as having reached remission and 6 patients $(14.0 \%)$ as having low disease activity, while SDAI identified 20 patients (46.5\%) as reaching remission and 12 patients (27.9\%) as having low disease activity. These data are illustrated in Fig. 1.

33 patients $(76.7 \%)$ had received treatment with NSAIDs before the first presentation, while 10 patients $(23.3 \%)$ didn't receive any treatment.

As a result of their first assessment, 40 patients (93.0\%) received treatment with MTX in doses ranging from $5 \mathrm{mg} /$ week to $20 \mathrm{mg} /$ week, with a mean dose of $11.16 \pm 4.47 \mathrm{mg} /$ week. (Fig. 2) The majority of patients (36 patients $-83.7 \%$ ) was receiving MTX ranging from 10 to $15 \mathrm{mg} /$ week. Three patients $(7.0 \%)$ didn't receive MTX, nor any other DMARD, because they presented with mild forms of disease (low joint counts), while 3 patients (7\%) received $20 \mathrm{mg} /$ week of MTX. Twenty-six patients
$(60.5 \%)$ received CS in different doses and forms of administration: 22 patients $(51.2 \%)$ were receiving Prednisone, 3 patients (7.0\%) received Betamethasone intra-articular and 1 patient received pulsetherapy with Methylprednisolone.

\section{Follow-up at 12 months}

At 12 months all 43 patients were receiving treatment with MTX in doses ranging from $5 \mathrm{mg} /$ week to $20 \mathrm{mg} /$ week, with a mean dose of $13.83 \pm 4.85 \mathrm{mg} /$ week (Fig. 3). The majority of patients (38 patients $-88.4 \%$ ) were receiving MTX in doses ranging from 10 to $20 \mathrm{mg}$ /week. Only 8 patients (18.6\%) were still receiving CS, all in the form of low-dose Prednisone (Fig. 4). All the patients who were receiving Prednisone at 12 months had started it at baseline and, although they had all reached remission or LDA, they continued low-dose oral CS as recommended by their attending rheumatologist.

\section{Comparisons between different treatment strategies}

After 12 months, there was no significant difference in terms of DAS28 and SDAI change since baseline between patients who received MTX in association with CS $(n=8)$ and those who received MTX monotherapy $(n=35)(p>0.05$ by the Wilcoxon signed-rank test). Similarly, there was no significant difference in DAS28 and SDAI change since baseline between patients who received short-term lowdose CS $(n=22)$ and those who received intra-articular CS or intra-muscular CS $(n=3)(p>0.05)$.

\section{DISCUSSION AND CONCLUSIONS}

In this study we followed up a cohort of patients with ERA attending our EIA research center between 2010-2014, in whom treatment was decided by the attending rheumatologist without having to respect a strict algorithm. Only patients naïve to DMARDS, who received at baseline treatment with MTX associated or not with CS and who fulfilled the classification criteria for RA were enrolled.

There are several particularities of the cohort at the first presentation worth to be discussed. First, the cohort had a remarkably short disease duration at baseline: despite the small number of patients, the median duration of symptoms at the first presentation (with a median of 8 weeks) was shorter than that reported in the Leiden EIA cohort (18.7 weeks) and also than that reported in the ESPOIR cohort (21.3 

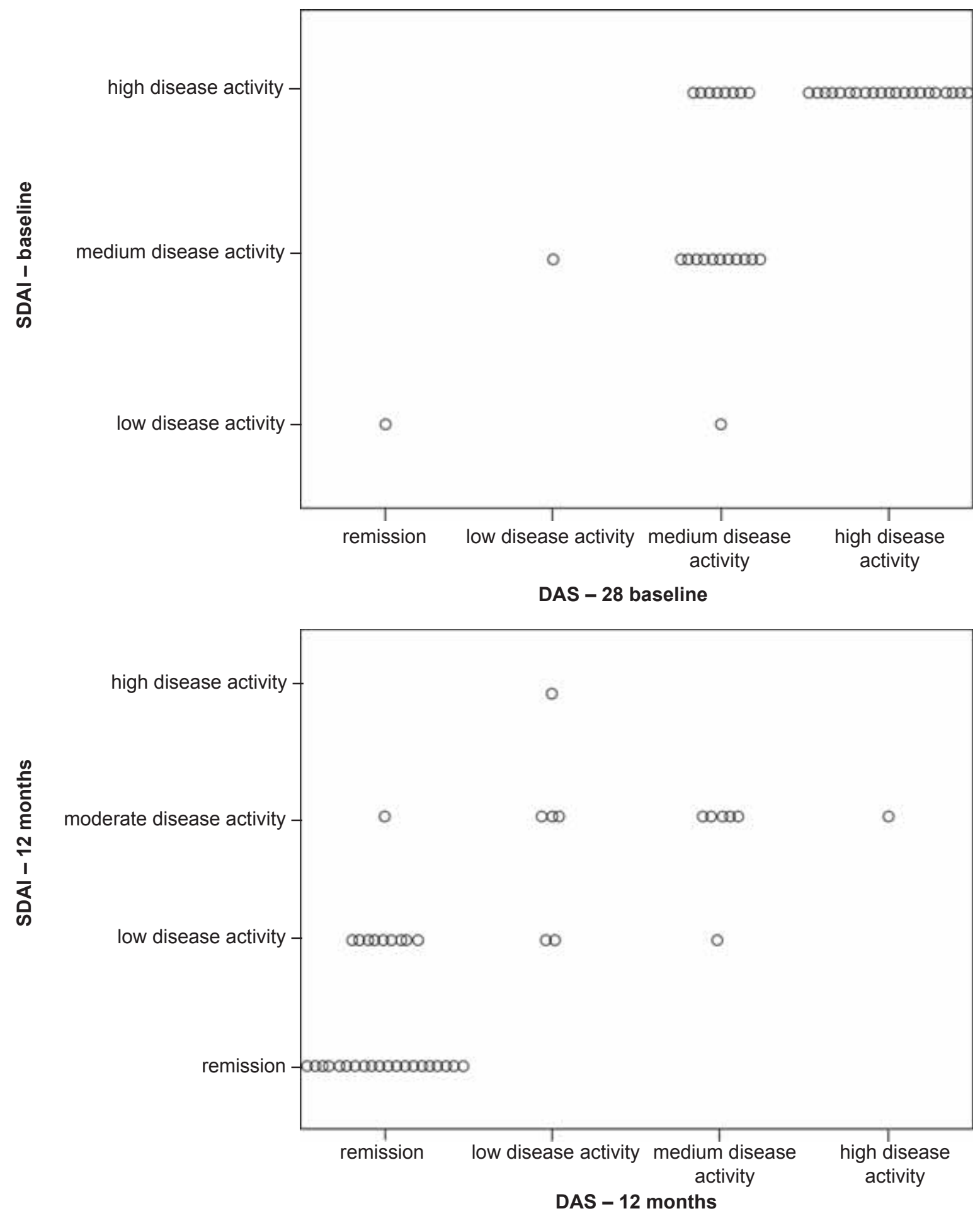

FIGURE 1. Correlations between DAS28 and SDAl at baseline and after 12 months, in a group of 43 patients with ERA

weeks). (2) Secondly, the percentages of patients positive for RF $(90.7 \%)$ and for ACPA $(90.7 \%)$ were higher than those reported in the Leiden cohort, where $58.7 \%$ of patients were positive for RF and $52.4 \%$ were positive for ACPA. (2) Also, the median value of ESR was lower in our cohort $(27 \mathrm{~mm} / 1 \mathrm{~h})$ than in the Leiden cohort $(31 \mathrm{~mm} / 1 \mathrm{~h})$, but higher than that reported in the ESPOIR cohort $(24 \mathrm{~mm} /$ 1h). (2) Thirdly, the values of DAS28 (4.89 \pm 1.03$)$ and SDAI $(27.96 \pm 10.10)$ were lower than those reported by Aletaha et al. for patients with ERA, in their study DAS28 having a mean value of $6.4 \pm 1.0$, while SDAI had a median value of $43.1 \pm 14.2$. (11).

As expected, all the parameters reflecting disease activity, as well as the serum level of RF and ACPA, decreased significantly under treatment during the 


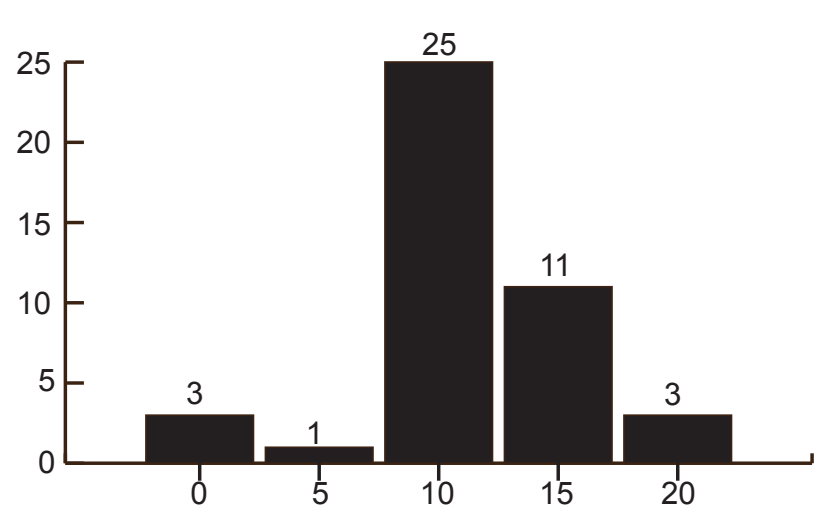

FIGURE 2. Distribution of MTX doses at baseline

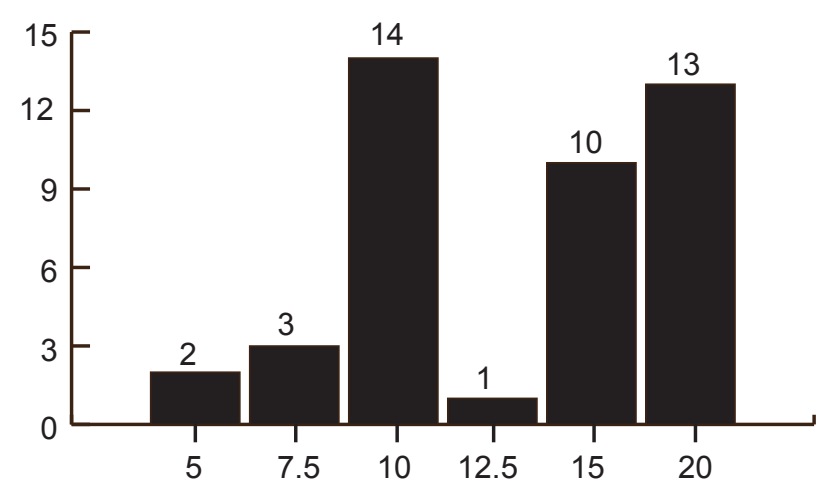

FIGURE 3. Distribution of MTX doses at 12 months

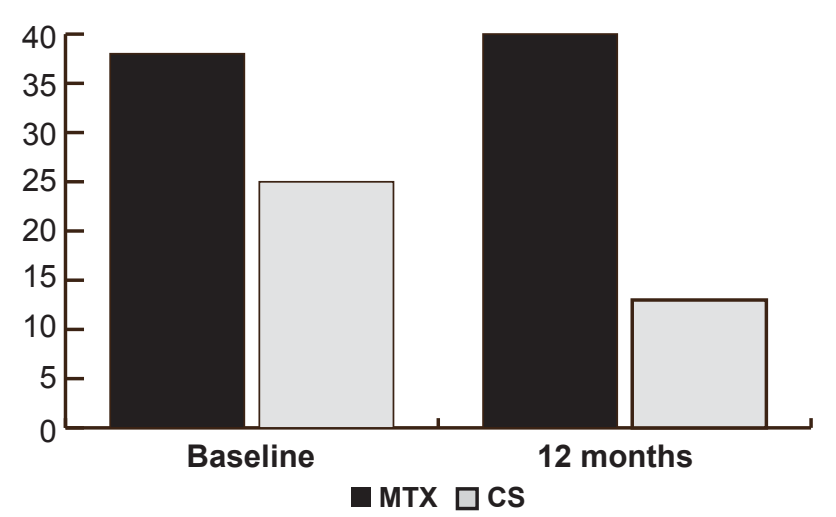

FIGURE 4. Distribution of MTX and CS treatment at baseline and after 12 months

study. The majority of patients reached remission or low disease activity at 12 months. SDAI identified more patients with moderate and high disease activity than DAS28 at baseline and less patients in remission or with low disease activity after 12 months of treatment, suggesting that SDAI is more rigorous than DAS28 in evaluating disease activity. Uhlig et al. showed that DAS28 identified twice as often remission as ACR/EULAR Boolean, CDAI and SDAI. (12)

There were noticeable differences in treatment between our cohort and other EIA or ERA cohorts.
Significantly more patients $(93.0 \%)$ received treatment with MTX after the first evaluation, than those in the Leiden cohort (76.3\%) or in the ESPOIR cohort $(67.0 \%)$. This can be explained by the fact that in the Leiden and ESPOIR cohorts, beside MTX, other DMARDs were used. An important element is the fact that, at 12 months, all the patients in our cohort were receiving MTX and the mean dose was higher than that prescribed at baseline. While the number of patients who received MTX increased during the study, the number of patients who received CS decreased significantly. No significant differences were noticed at 12 months between patients who received at baseline MTX associated with CS and those who received MTX monotherapy.

Another observation regarding CS is that at 12 months all patients still receiving CS were taking only low-dose oral CS. This is probably due to the fact that MTX therapy had reached its maximum efficacy level and disease activity was controlled.

There was no significant difference regarding outcomes at 12 months between patients who received low-dose Prednisone and those who received intra-articular CS. This results are in line with the ACR recommendations on the management of RA but, at this point, there are no available data about the structural effect of intra-articular CS. Further studies are necessary to prove that intra-articular CS are as effective in preventing radiologic progression as low-dose oral CS.

An important element is the fact that disease activity was influenced by the initiation of treatment with MTX at baseline.

The main limitation of our study is the small number of patients. Also, assumptions on treatment efficacy are biased by the fact that treatment decisions were adapted to each patient.

In conclusion, our study highlights once again the importance of prompt initiation of MTX in patients with ERA, leading to improved clinical and laboratory outcomes after 12 months of follow-up. The fact that there is no significant difference regarding long-term outcomes between patients who receive low-dose Prednisone and those who receive intra-articular CS, recommends the latter version as frequently as possible. SDAI seems to be superior to DAS28 in evaluating disease activity by its capacity to identify higher levels of disease activity in more patients than DAS28 does. 


\section{REFERENCES}

1. Boers $\mathbf{M}$. Understanding the window of opportunity concept in early rheumatoid arthritis. Arthritis Rheum. 2003; 48: 1771-1774.

2. van Nies J.A.B., Tsonaka R., Gaujoux-Viala C., et al. Evaluating relationships between symptom duration and persistence of rheumatoid arthritis: does a window of opportunity exist? Results on the Leiden Early Arthritis Clinic and ESPOIR cohorts. Ann Rheum Dis. 2015;74: 806-812.

3. Smolen J.S., Landewe R., Breedveld F.C., et al. EULAR recommendations for the management of rheumatoid arthritis with synthetic and biological disease-modifying antirheumatic drugs: 2013 update. Ann Rheum Dis. 2014; 73: 492-509.

4. Singh J.A., Saag K.G., Bridges S.L., et al. 2015 American College of Rheumatology Guideline for the Treatment of Rheumatoid Arthritis. Arthritis\&Rheumatology; 2016; 68: 1-26.

5. Schoels M., Knevel R., Aletaha D., et al. Evidence for treating rheumatoid arthritis to target: results of a systematic literature search. Ann Rheum Dis. 2010; 69: 638-643.

6. Smolen J.S., Aletaha D., Bijlsma J.W., et al. Treating rheumatoid arthritis to target: recommendations of an international task force. Ann Rheum Dis. 2010; 69: 631-637.

7. Bykerk V.P., Massarotti E.M. The new ACR/EULAR remission criteria: rationale for developing new criteria for remission. Rheumatology 2012; 51: vi16-vi20.
8. Aletaha D., Neogi T., Silman A.J., et al. 2010 Rheumatoid arthritis classification criteria: an American College of Rheumatology/ European League Against Rheumatism collaborative initiative. Arthritis Rheum. 2010; 62: 2569-2581.

9. Prevoo M.L.L., van ,t Hof M.A., Kuper H.H., et al. Modified disease activity scores that include twenty-eight-joint counts: development and validation in a prospective longitudinal study of patients with rheumatoid arthritis. Arthritis Rheum 1995; 38: 44-48.

10. Aletaha D., Ward M.M., Machold K.P., et al. Remission and active disease in rheumatoid arthritis: defining criteria for disease activity states. Arthritis Rheum 2005; 52: 2625-2636.

11. Aletaha D., Funovits J., Keystone E.C., Smolen J.S. Disease activity early in the course of treatment predicts response to therapy after one year in rheumatoid arthritis patients. Arthritis Rheum. 2007; 56: 3226-3235.

12. Uhlig T., Lie E., Norvang 1, et al. Achievement of Remission and Low Disease Activity Definitions in Patients with Rheumatoid Arthritis in Clinical Practice: Results from the NOR-DMARD Study. J Rheumatol.: jrheum.151132. 2016 [Epub ahead of print] 